\title{
DESIGN AND VERIFICATION OF REMOTE SENSING IMAGE DATA CENTER STORAGE ARCHITECTURE BASED ON HADOOP
}

\author{
Dejin Tang ${ }^{1}$, Xiaoming Zhou ${ }^{2}$, Yang Jing ${ }^{1}$, Wang Cong ${ }^{1}$, Caiping $\mathrm{Li}^{2}{ }^{\text {* }}$ \\ ${ }^{1}$ National Geomatics Center of China, 100830,28\#Lianhuachi West Road, BeiJing, China -tangdejin@ @ ngcc.cn \\ ${ }^{2}$ Beijing Institute of Remote Sensing Information,100192, Haidian District ,Beijing,China-zxm2913@163.com
}

Commission III, WG III/6

KEY WORDS: Data center, Storage strategy, Parallel processing, Hadoop, HBase

\begin{abstract}
:
The data center is a new concept of data processing and application proposed in recent years. It is a new method of processing technologies based on data, parallel computing, and compatibility with different hardware clusters. While optimizing the data storage management structure, it fully utilizes cluster resource computing nodes and improves the efficiency of data parallel application. This paper used mature Hadoop technology to build a large-scale distributed image management architecture for remote sensing imagery. Using MapReduce parallel processing technology, it called many computing nodes to process image storage blocks and pyramids in the background to improve the efficiency of image reading and application and sovled the need for concurrent multi-user high-speed access to remotely sensed data. It verified the rationality, reliability and superiority of the system design by testing the storage efficiency of different image data and multi-users and analyzing the distributed storage architecture to improve the application efficiency of remote sensing images through building an actual Hadoop service system.
\end{abstract}

\section{INTRODUCTION}

With the great-leap-forward development of remote sensing image acquisition ability, ground remote sensing system is faced with a bottleneck problem which is about the processing and interpretation and sharing of huge data. Traditional data storage strategy is usually enslaved to the disk performance and bandwidth. It is far from enough to satisfy the timeliness requirements of the existing multi-user cooperative application. Meanwhile the image information generated by large data cannot be interpreted and processed by the simply stacking and updating of hardware. The new technology and processing strategy is needed to solve the problem of processing capacity of existing systems.Focusing on big data, a number of emerging data storage, data processing, data mining and analysis technologies have emerged, such as cloud computing, NoSQL, CloudDB, and distributed system infrastructure hadoop developed by the Apache Foundation. The above technologies and platforms make mass data processing more convenient, cheaper, and faster. Currently, it has been applied in ecommerce, mobile communications, and intelligent transportation.

The data center is a new concept of data processing and application proposed in recent years. Its main part is data. It supports parallel computing and is compatible with different hardware cluster. It can increase the efficiency of data parallel applications through optimizing the data storage management structure and fully using the cluster resource computing nodes. The data center processing technology is based on the optimization design of data storage management system, which can fully call all storage node devices and computing resources, establish a high-speed parallel processing mechanism in the background, and realize the optimization strategy of massive data processing. At present in the data storage scheme, hadoop is widely used in architecture design. It is proved to have natural advantages in processing the storage management of structured multi-source heterogeneous mass data. But it is not proved that there is a greater efficiency improvement in the actual production system for a large number of unstructured image data.

\section{HADOOP PROJECT DESIGN CONCEPT}

Hadoop is an open source distributed system infrastructure developed by the Apache foundation. The architecture can develop distributed programs without knowing the details of the underlying details, and make full use of the power of the cluster to carry out high-speed computation and storage. Hadoop is made up of many elements, at the bottom of which is Hadoop Distributed File System (HDFS), which stores all the files of all storage nodes in the Hadoop cluster. The upper layer of HDFS is the MapReduce engine. Through the establishment of the core distributed file system HDFS and MapReduce processing process of Hadoop distributed computing platform, and the establishment of data warehouse attack Hive and distributed database Hbase, it basically covers all the core technologies of Hadoop distributed platform.

Applying Hadoop processing technology to remote sensing image organization and management, combined with data center construction ideas, the architecture of Hadoop technology in this paper is illustrated in Figure 1.

\footnotetext{
"National Key R\&D Program of China (No.2017YFB0503700) tangdejin@ngcc.cn;phone:18911938487
} 


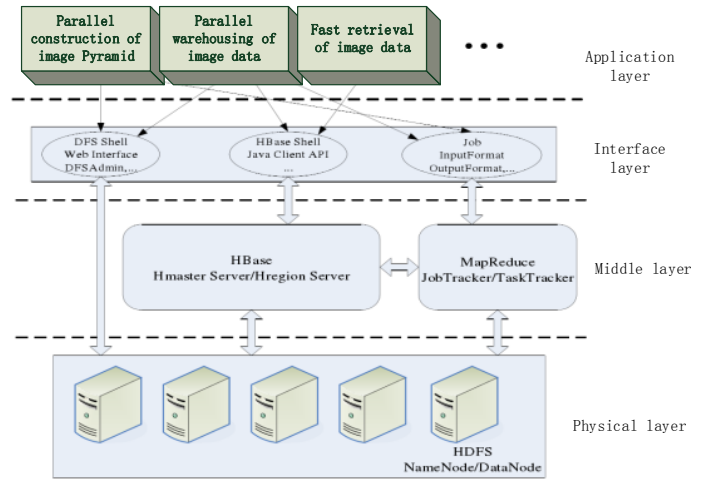

Figure 1 Hadoop technical architecture diagram

\subsection{Distributed storage}

The definition of large-scale distributed storage system is as follows: "distributed storage system is a large number of ordinary PC servers that provide storage services as a whole through network interconnection." Distributed storage system is a system that collectively works together with different storage devices on different nodes in the network by distributed technology, and provides data storage and business access functions together.

The most important feature of distributed storage system is that data is stored on each independent node of the distributed storage system for transparent access by users. Distributed storage system adopts extensible system structure. It uses multiple storage servers to share storage load and location servers to locate and store information. It not only improves the reliability, availability and access efficiency of the system, but also can be easy to extend.

HDFS (Hadoop Distributed File System) is a distributed file system designed to hold a large amount of data (PB or EB) and provides quick access to these data. Data is stored on multiple machines in a redundant manner, saving fault tolerance for failures and high availability of parallel applications.

HDFS is similar to the existing network file system. It is a distributed network file system running on the ordinary hardware. However, it is also different from the common network file system. HDFS has high fault tolerance and can be deployed on low-cost hardware. At the same time, HDFS relaxes the demand for POSIX, so that it can access the file data in the form of flow, providing high-throughput of application data. It is suitable for large data sets of applications.

Compared with the network file system, the design of HDFS has the following characteristics: high performance, high reliability, high availability and high scalability.

- HFDS is designed to preserve very large amount of data information, and it is necessary to distribute data to a large number of machines.

- HFDS data preservation is reliable, and data is still available if some of the machines in the cluster are not working properly.

- By simply adding some machines, it provides fast, scalable data access capabilities that enable HDFS to serve more clients

- The applications on HDFS are different from the general applications. They are mainly streaming read and batch processing. Compared to the low latency of data access, the key is the high throughput of data access.

- HDFS and Hadoop MapReduce can be integrated well, and in a possible case, can make data read and compute localisation.

\subsection{MapReduce Parallel processing model}

MapReduce is a programming model for data processing, which is essentially parallel. Its advantage lies in dealing with large data sets. This is different from MPI, which is suitable for computing intensive applications. MapReduce is made up of two verbs, Map and Reduce. "Map (deployment, mapping)" is to decompose a task into multiple tasks. "Reduce" is the result of multitask processing after decomposition, and the final analysis result is obtained. No matter in the real society, or in the program design, a work can be split into a number of tasks. The relationship between tasks can be divided into two types: one is not related to the task which can be executed in parallel; the other is a mutual dependence between tasks, the order can not be reversed, such tasks cannot be handled in parallel . In a distributed system, the machine cluster has many nodes, split the tasks which can be processed parallelized, and then processing each idle machine node, which can greatly improve the computational efficiency. At the same time, this kind of resources independence will undoubtedly provide the best design assurance for the expansion of the computing cluster . After the task is decomposed, it need to reorganize the results after processing. This is what Reduce wants to do.

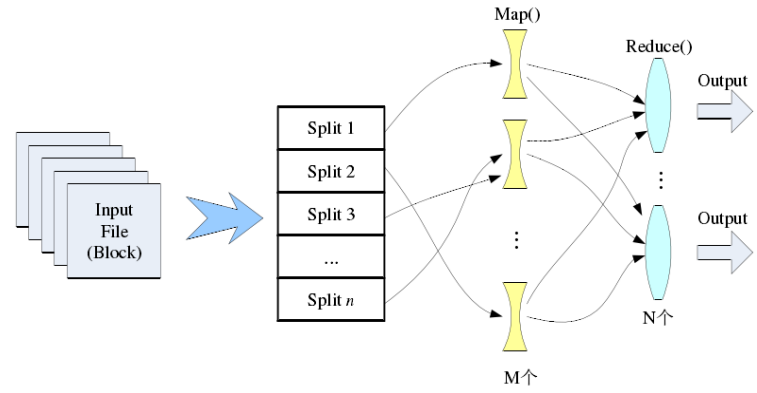

Figure 2 MapReduce schematic diagram

\subsection{Hbase database architecture}

Hbase is part of Hadoop, and is an open source, distributed database based on the column storage model . The purpose of Hbase is to solve a series of problems encountered by traditional relational databases when processing massive data. In traditional relational databases, such as Mysql, users usually need to ensure data consistency when designing with these databases, but they are far from perfect in database development and reliability. The design of Hbase is generated in the background of data developing towards PB and TB level. By realizing the storage and reading and writing of large data, a large number of users can get a haircut visit. Hbase regards HDFS as its file storage system, and realizes the parallel warehousing of massive data through MapReduce. The specific architecture diagram is shown in Figure 3. 


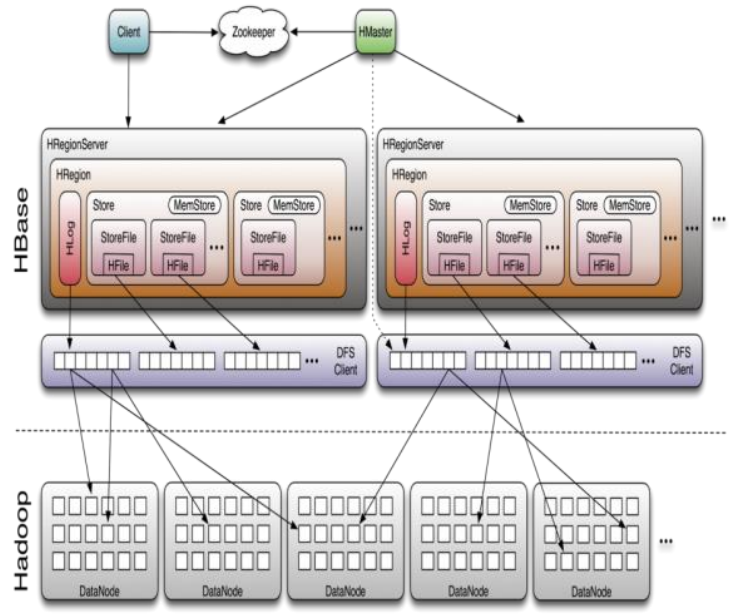

Figure 3 Hbase architecture diagram

\section{THE STORAGE STRUCTURE OF REMOTE SENSING IMAGE DATA CENTER}

\subsection{Construction of remote sensing image HDFS}

The simulation data center application system is used as the master node of the Namenode. Meanwhile, the other application systems or storage devices are set up as HDFS DataNode nodes, and a virtual HDFS system is simulated. The entire HDFS file system is set as a data sharing pool of remote sensing images. All data application services are invoked by the center. The DataNode of each subsystem and other storage nodes are used as the actual carrier of image data.

The data process is: the data center receives the data processing completion reports and the data cataloging informations, and then initiates the data downloading procedure. Finally,it stores the completed primary and secondary remote sensing product data in the internal HDFS nodes, the catalogs the data and other metadata information on the NameNode. After the data storage is completed, the Pyramid image construction is started. The metadata information that will complete the result also exists on the NameNode.

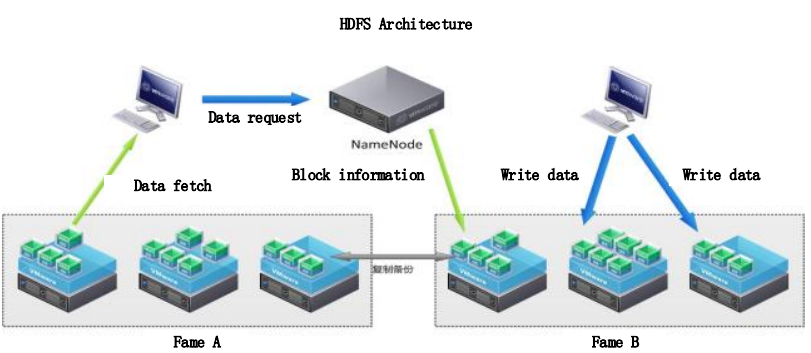

Figure 4 HDFS architecture diagram

\subsection{Image database establishment}

The distributed image data center network relies on the internal backbone network of remote sensing image production system. Each application client interprets and interprets the image information by calling the data center, at the same time, it can also access historical images to compare and perform intelligence information.

The whole system architecture is built with HDFS's distributed storage system.
(1)NameNode is deployed in the data center system server machine room, and uses a high performance server. At the same time, in order to improve the availability of NameNode, double machine is used. On the same cabinet, several application servers are selected to set up and deploy multiple DataNode for centralized management, and dynamically expand according to the subsequent data scale.

(2)On the five adjacent cabinets, more than one DataNode are deployed respectively as an entity data storage pool for image data.

(3) Each terminal operates and allocates different rackids. The HDFS rack-aware feature is mainly used. When the requester queries the data center's NameNode and locates the DataNode node of the organization to extract files, it will preferentially deploy the DataNode locally. That is, reads are performed on the document storage pool, rather than extracting data from the DataNode on the switch to other cabinets.

The image data center solution centrally stores and publishes satellite images of terrestrial application systems. Each application terminal accesses the data center through the network to achieve the purpose of sharing and exchanging image data. The simulated data center general flow is as follows:

- Each ground subsystem sends image data and control information directly to the data center.

- Each application terminal workstation inquires and extracts the required images through data center, and data center provides registration, publication, query and extraction services of the whole region image.

The core idea of the shared exchange technology framework of image data center is the distributed storage of entity data, centralized storage of metadata such as image document index, etc.

The data sharing and exchange schematic of the entire data center is as follows:

Data sharing and exchange schematic diagram

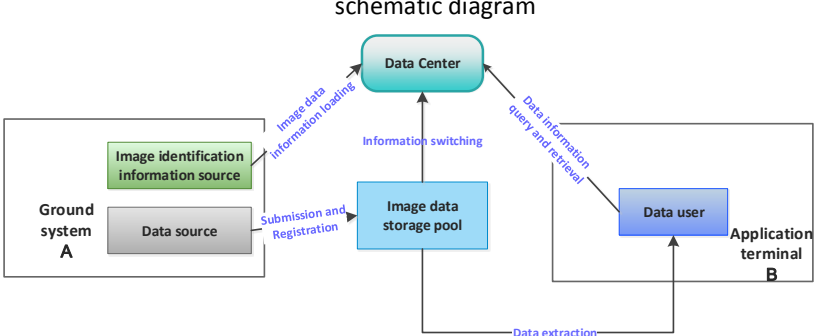

Figure 5 Shared exchange schematic

Through the distributed deployment, each image application subsystem can deploy and manage its own document storage pool. The data center does not need to accommodate all the image data. Furthermore, when the application terminal queries the data center for data extraction, it is directed to its own management. When nodes extract data, they do not need to connect to the data center through the network switch, and can achieve the highest network performance. In this way, the data center's communication burden can be greatly reduced, ensuring the reliability and availability of the entire system. The data flow of the entire system is shown below. 


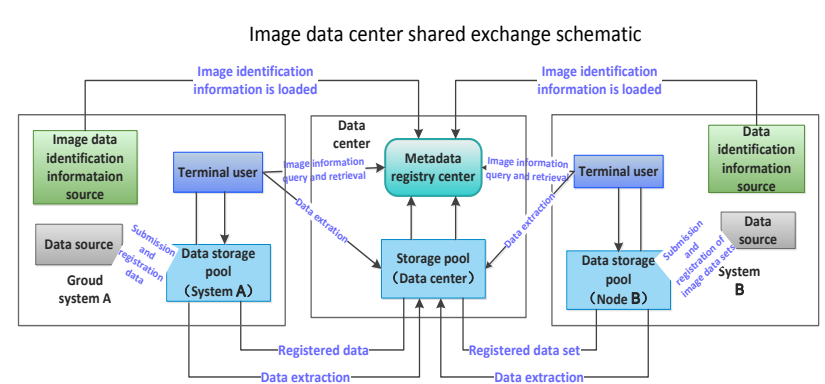

Figure 6 Flow diagram

\section{TEST VERIFICATION}

The technical scheme in this paper were used in the actual production systems. The traditional image disk array and application server are used as the hardware basis. The total disk array capacity is $80 \mathrm{~TB}, 5$ DataNode nodes are set up, and the full-range of image of a certain area is stored at the same time. The image resolution is better than 1 meters. The details are as follows:

(1) Data storage processing efficiency

A workstation (D30) operates for the user client and writes data to the disk array, compares the efficiency of FTP and Hadoop, and takes 1TB image data as a comparison. The results are as follows. It should be noted that FTP only completed the simple transmission and storage of images. Hadoop also completed parallel computing while data transmission and storage, and built image pyramids, data blocks, and redundant backups. This work mode is continuous parallel work. In order to reflect the processing efficiency of large data volume, so take 1TB image processing results for comparison.

\begin{tabular}{c|c|c}
\hline $\begin{array}{c}\text { Data writing } \\
\text { method }\end{array}$ & $\begin{array}{c}\text { Write data } \\
\text { volume }\end{array}$ & $\begin{array}{c}\text { Read speed MB/S } \\
\text { (take the average) }\end{array}$ \\
\hline \multirow{2y}{*}{ FTP } & \multirow{2}{*}{$1 \mathrm{~TB}$} & 10.7 \\
\cline { 1 - 1 } Hadoop & & 10.9 \\
\hline
\end{tabular}

Table 1 Efficiency analysis table

(2) Data reading efficiency

The operation of 10 sets of the same configuration of the client workstation (D30) were operated both in single-reading and multi-reading way for comparition, and used FTP and Hadoop to read the efficiency of the area images as analysis to read 100GB images. The average value of the data was used as a comparison (10GB connection for network switching equipment).

\begin{tabular}{c|c|c|c}
\hline $\begin{array}{c}\text { Data } \\
\text { reading } \\
\text { method }\end{array}$ & $\begin{array}{c}\text { Reading } \\
\text { data } \\
\text { volume }\end{array}$ & $\begin{array}{l}\text { Stand-alone read } \\
\text { speed MB/S(take } \\
\text { the average) }\end{array}$ & $\begin{array}{c}10 \text { clients read } \\
\text { speed MB/S }\end{array}$ \\
\hline FTP & \multirow{2}{*}{$100 \mathrm{~GB}$} & 11.43 & 8.43 \\
\cline { 3 - 4 } Hadoop & & 82.68 & 84.31 \\
\hline
\end{tabular}

Table 2 Efficiency analysis table

\section{CONCLUSION}

This paper established a new distributed image storage management system. It used the mature Hadoop technology, built the management architecture for distributed processing of massive remote sensing images, fully called all storage nodes through the MapReduce parallel processing technology and resource optimization strategy, completed the operation of image data writing, computing, storage and blocking, and building Pyramid and stored in the in the Hbase database. It could analysis the advantage of Hadoop technology in image management though the actual data storage and reading test.

Experiments proved that the distributed storage system built by Hadoop was much better than the traditional disk storage structure for the storage and reading efficiency of the single file. And the greater the amount of data, the higher the efficiency was. The system architecture could fully invoke the built image processing equipment of the space remote sensing ground processing system and use advanced large data processing technology to realize unified management and processing of remote sensing data. It also could maximize the use of hardware processing resources for cluster use to improve the data processing capabilities of the whole ground system.

\section{REFERENCES}

Yu Fenxiang, Wang Guangxia, Wang Gang. Rapid scheduling and display of remote sensing images with large amount of data. Science of Surveying and Mapping, 2006(3),pp. 30.

Zhang Jinfang, Hu Xiaohui, Zhang Hui, Wang Rui, Li Haichang. Analysis and application of large data information of satellite image. Big Data, 2016(9),pp. 20.

Peng Lei. Key technology research and system implementation of remote sensing image large data management system. Master's thesis of the University of Electronic Science and technology, 2014,pp. 134-135.

Li Xuebo. Storage and management of massive spatial data under Hadoop architecture. Master's thesis of Wuhan University, 2017,pp. 89-95.

Revised March 2018 